After a period of relative stability and deeper embedding, the debate about the role and function of the VLE (virtual learning environment) within the institution is gathering pace again. Many institutions in the UK are in the process of reviewing their current VLE provision in the light of changing pedagogical requirements, more administrative integration and the emergence of new classes of social media on the wider web.

In the past, the requirement for deeper integration with other (administrative) systems gave rise to the MLE (managed learning environment) concept. Later, the demand for greater personalisation and the availability of new web tools gave birth to the PLE (personal learning environment) debate, in which people radically reconceptualised the notion of a learning environment. During these phases, however, the VLE still remained a dominant force within institutions. This has resulted in a tension between the role of the VLE as a common tool for the institutional community, the desire to make it permeable to the institutional network and the wider web and to allow greater levels of personalization / customization for individuals and institutions.

A number of working solutions are now emerging to address these tensions. This briefing paper will explore five emerging models, discuss the strengths and weaknesses of each and link to working examples and further sources of information.

\section{JISC cetis}

centre for educational technology

\& interoperability standards

\section{Distributed Learning Environments}

\section{A Briefing Paper}

By Sheila MacNeill and Wilbert Kraan 


\section{DISTRIBUTED LEARNING ENVIRONMENTS}

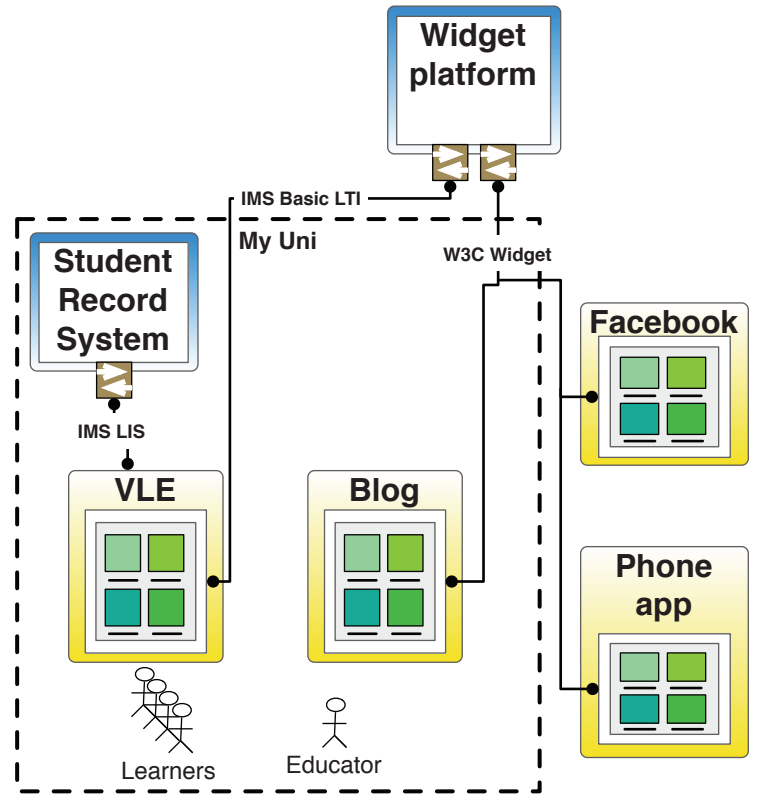

One system in the cloud, many outlets.

The core of this model is almost an inversion of the portal idea: a collection of services is gathered in one place, and from there broadcast to a range of platforms. The platforms range from an existing VLE to a smartphone application (app).

The diagram above illustrates one possible instantiation where a single hosting service 'in the cloud' serves a collection of educationally useful, standardised widgets to a VLE, a blog, a social network and a smartphone app. Possible variations of the model could be hosted by one institution, or a federation of institutions. Another interesting variation of this model, as exemplified by the plugjam system, gathers nearly all user facing functions of an institution, not just educational tools, and exposes them in the same way.

\section{EXAMPLES:}

- Apache Wookie (Incubating) Widget server, http://incubator.apache.org/wookie/

- Icodeon Common Cartridge platform, http://ccplatform.blogspot.com/

- Plugjam, http://wnw.plugjam.com/

\section{STRENGTHS:}

- Secure integration with current VLE system.

- Burgeoning developer community.

- Easy deployment on multiple platforms.

- Standards promise the ability to share widgets and widget instances across the sector (and beyond).

- Allows teachers to deploy the same content in many different locations e.g. a blog, social network site as well as the VLE.

WEAKNESSES:

- No standard way (yet) to feed user interaction from the central system back to other applications.

- Some competing standards, and one specifically educational one (IMS Basic Learning Tools Interoperability ) that has not been finalised just yet.

- Implementation experience is in the pilot phase.

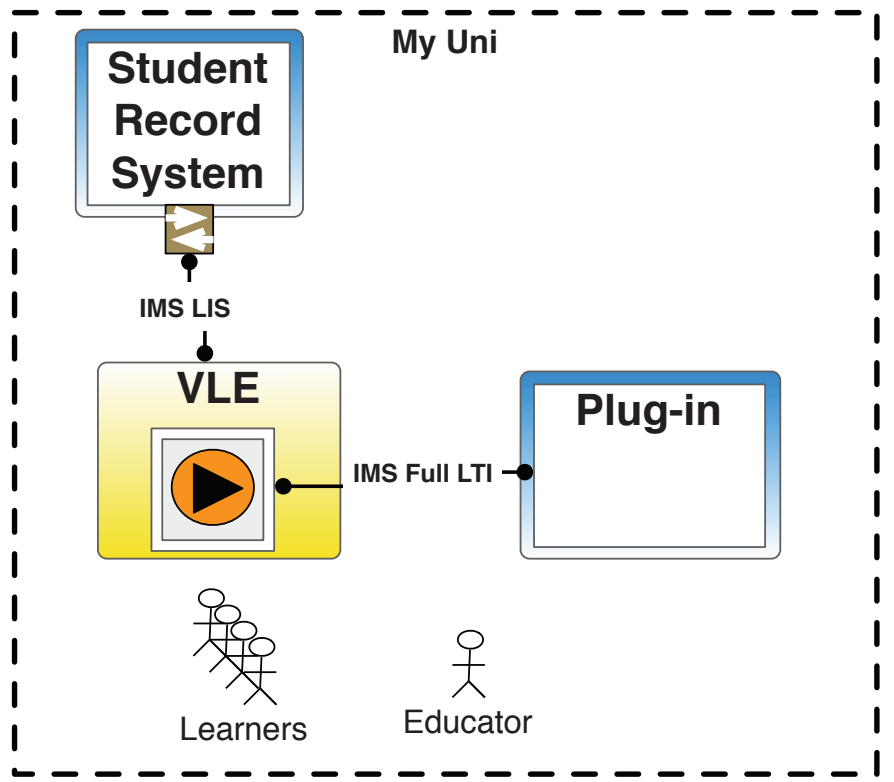

\section{Plug-ins to existing VLEs.}

This model is premised on the possibility to extend the functionality of an existing VLE with a plug-in; a dedicated piece of software that exists solely to provide additional features to a host system. Because the plug-in is tightly bound to the host system, it is possible for a VLE to launch the plug-in and provide rich user identity, course, role and other session information. It can also expect a lot of the same kind of information in return.

\section{EXAMPLES:}

- Icodeon SCORM Player, http://www.icodeon.com/product.html

- QTlengine, http://qtiengine.qtitools.org/

\section{STRENGTHS:}

- Rich interaction data flow between tool and VLE.

- Burgeoning developer community working with the major VLE systems that are used in HE sector.

- Potential to share many tools/plug-ins across the sector.

\section{WEAKNESSES:}

- Currently requires a deep dependency on the host VLE.

- IMS full Learning Tools Interoperability standard still under development.

- Not so suitable for pulling in social media from the wider web, or deployment of tools to platforms other than the major VLEs. 


\section{DISTRIBUTED LEARNING ENVIRONMENTS}

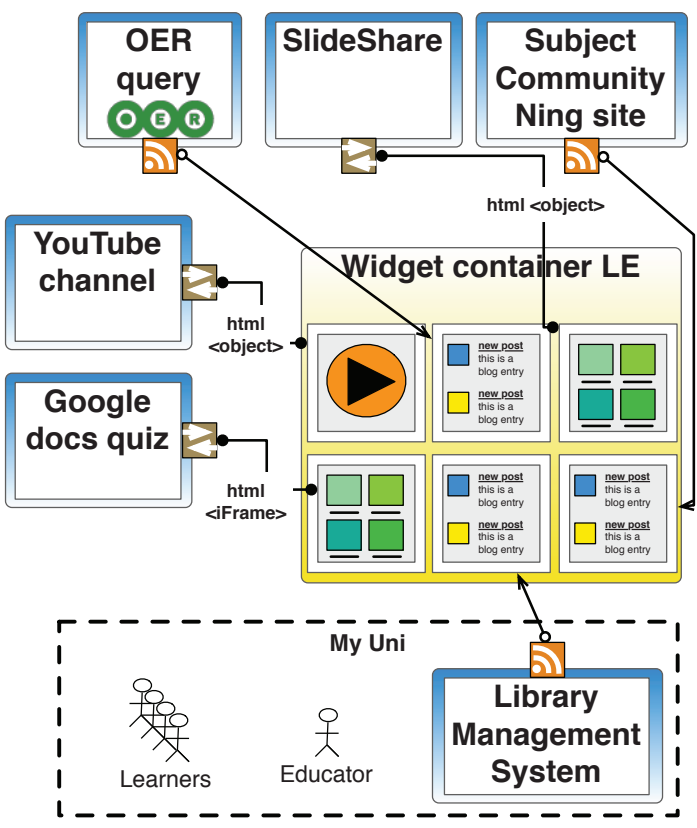

Many widgets from the web into one Widget container.

This model captures the by now classic social media case of easy mash-up of a variety of sources using nothing but Software as a Service applications. It is also one of the archetypal implementations of a Personal Learning Environment. As such, quite a bit of assembly is required even if no programming skills are needed for assembly or deployment.

This diagram illustrates a fairly typical case where the services that go into the widget container learning environment are all relatively static. The set-up is perfectly achievable now, but lacks interactive, collaborative features such as discussion forums.

\section{EXAMPLES:}

- Pageflakes, http://wnw.pageflakes.com/

- Netvibes, http://www.netvibes.com/

- iGoogle, http://www.google.com/ig

- wetpaint, http://www.wetpaint.com/

\section{STRENGTHS:}

- Relatively easy and quick to implement (no deep coding skills required).

- Demonstrates the principles and potential for aggregating disparate resources into one collective space.

- Low cost; uses mainly freely available tools/services.

\section{WEAKNESSES:}

- Not integrated with institutional systems (although can provide access to available institutional data e.g. institutional RSS feeds).

- Little scope to capture interaction data.

- Limited integration of any service that requires authentication.

- Lack of durability; potential for free services to change to pay for model with little or no warning.

- Only for the technically confident and tolerant.

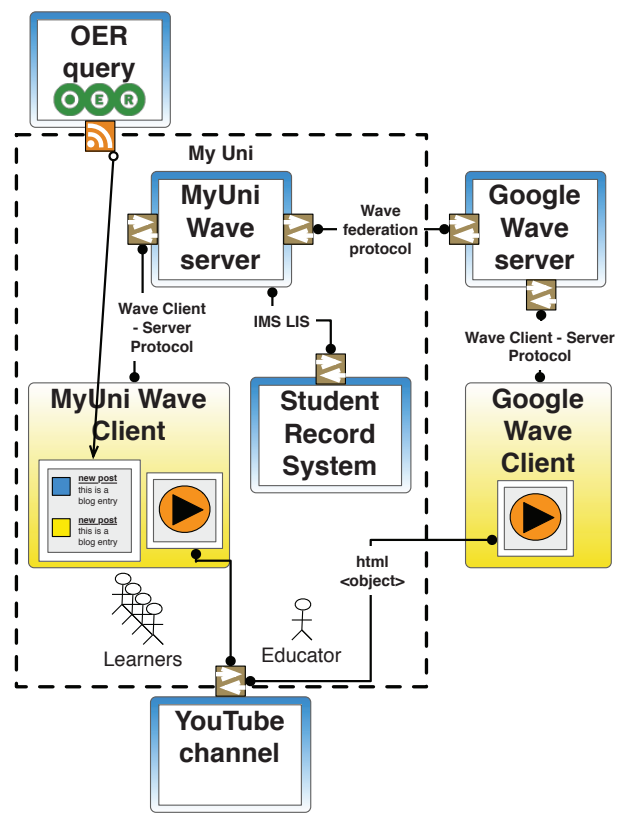

Many providers and many clients.

As with email, it is possible to federate an infrastructure out of many similar clients and servers. Google Wave is a recent, mainstream example of this type of infrastructure. A message or 'Wave' can be started by one person in one client, and replicated via public and private servers to the users of other clients. In this way, it is possible to create a synchronous, highly interactive collaborative workspace (wave). In addition, technologies such as Google Wave now allow other widgets and services to be integrated into their messages (waves), as well their waves to be embedded in other web platforms.

\section{EXAMPLES:}

- Email.

- Google Wave, http://wave.google.com

\section{STRENGTHS:}

- Highly interactive.

- Fine balance of control; smaller dependency on a single vendor.

- Truly collaborative.

- Flexible.

\section{WEAKNESSES:}

- Technology is relatively new.

- Significant unsolved usability problems. 


\section{DISTRIBUTED LEARNING ENVIRONMENTS}

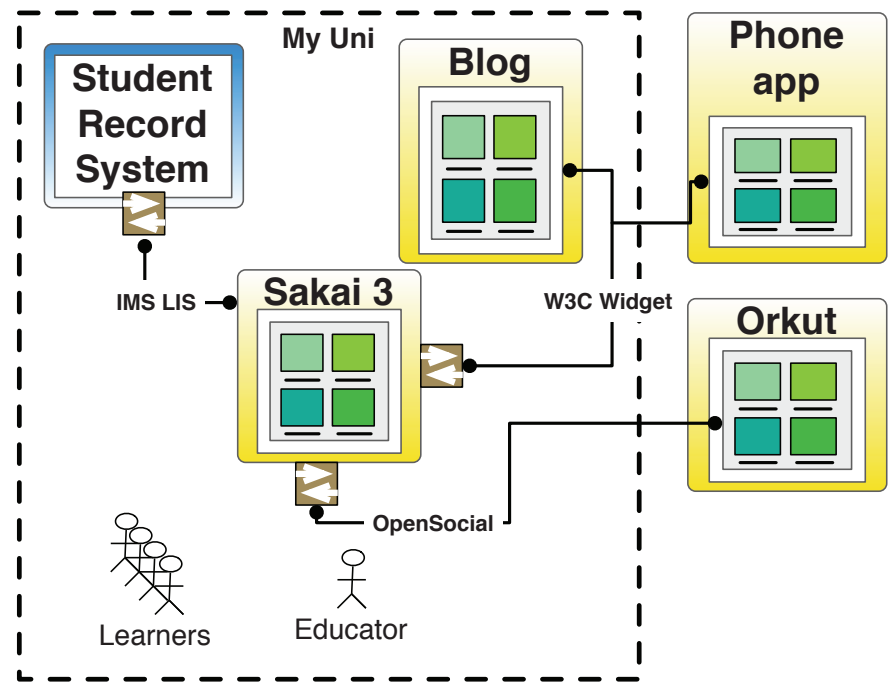

Both a provider and a client.

Where the 'many providers and many clients' model relied on a level of indirection, the 'both a provider and a client' model does the provisioning and consumption directly, and to equal degree. Ideally this model ought to allow the seamless import of functions and information from systems that are dedicated to those functions and that information, while allowing other systems to easily make use of those things the system itself specialises in. In that sense, the approach resembles the ideal, if not the method, of Service Oriented Architecture (SOA).

In this particular illustration, Sakai 3 exposes some of its content via W3C widgets to a blog and a smartphone app, and some of its functions to a social networking site.

\section{EXAMPLES:}

- Sakai 3, http://sakaiproject.org/

\section{STRENGTHS:}

- Lets every system play to its' strengths.

- Truly distributed architecture, so balanced locus of control.

- Many options for sharing functionality across the sector.

- Flexible.

\section{WEAKNESSES:}

- Not many implementations yet.

- Heavy load on the combined provider and client.

- Potential single point of failure.
FURTHER READING:

Composing your learning environment, new models, JISC CETIS 2009 Conference

http://wiki.cetis.ac.uk/Composing_your_Learning_

Environment\%3B_new_models

\section{IMS LTI (Learning Technology Interoperability)}

http://www.imsglobal.org/toolsinteroperability2.cfm

\section{W3C Widgets}

http://www.w3.org/TR/widgets/

\section{Wikipedia Google Wave entry}

http://en.wikipedia.org/wiki/Google_wave

\section{Aspect IMS Common Cartridge \& SCORM demonstrator}

http://aspect.eun. org/node/40

\section{Google Wave and Teaching and Learning blog post} http://blogs.cetis.ac.uk/wilbert/2009/05/29/google-waveand-teaching-learning/

\section{Google wave 101, MASHe blog post}

http://www.rsc-ne-scotland . org . uk/mashe/2009/11/googlewave-101-presentations-from-scottish-vle-groups/

\section{The headless VLE blog post}

http://blogs. cetis.ac. uk/sheilamacneill/2009/11/13/theheadless-vle-and-other-approaches-to-composing-learningenvironments/

Background image: (C) Award Creative, 2009. Image courtesy of JISC.

This work is licensed under a Creative Commons Attribution-NonCommercialShareAlike 2.5 License (http://creativecommons.org/licenses/by-nc-sa/2.5/) and adheres to the JISC CETIS publication Policy

(http://wiki.cetis.ac.uk/JISC_CETIS_Publication_Policy). 